\title{
CANONICAL TIME-FREQUENCY, TIME-SCALE, AND FREQUENCY-SCALE REPRESENTATIONS OF TIME-VARYING CHANNELS*
}

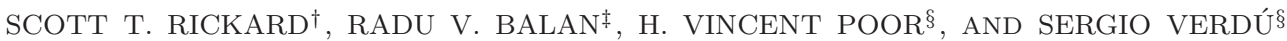

\begin{abstract}
Mobile communication channels are often modeled as linear time-varying filters or, equivalently, as time-frequency integral operators with finite support in time and frequency. Such a characterization inherently assumes the signals are narrowband and may not be appropriate for wideband signals. In this paper time-scale characterizations are examined that are useful in wideband time-varying channels, for which a time-scale integral operator is physically justifiable. A review of these time-frequency and time-scale characterizations is presented. Both the time-frequency and time-scale integral operators have a two-dimensional discrete characterization which motivates the design of time-frequency or time-scale rake receivers. These receivers have taps for both time and frequency (or time and scale) shifts of the transmitted signal. A general theory of these characterizations which generates, as specific cases, the discrete time-frequency and time-scale models is presented here. The interpretation of these models, namely, that they can be seen to arise from processing assumptions on the transmit and receive waveforms is discussed. Out of this discussion a third model arises: a frequency-scale continuous channel model with an associated discrete frequency-scale characterization.
\end{abstract}

Key words: Time-Frequency, Time-Scale, Frequency-Scale, Delay, Doppler, Dilation, Doubly Spread, Time-Varying, Canonical Channel Models, Rake Receiver, Wideband Regime

1. Introduction. It is common to assume that a received communication signal is composed of superpositions of different versions of the transmitted signal. These different versions arise from reflections of the signal off of scatterers in the environment. In the time-scale channel model, each reflection is a delayed and time scaled copy of the transmitted signal. The delays arise from differing path lengths from transmitter to scatterer to receiver. Relative motion of the transmitter, scatterers, or receiver causes time dilations/contractions of the transmitted waveform $x(t)$. Thus, each reflection is of the form,

$$
x_{a, b}(t)=\frac{1}{\sqrt{|a|}} x\left(\frac{t-b}{a}\right)
$$

* This research was supported in part by the Office of Naval Research under Grant N00014-03-10102 .

†School of Electrical, Electronic and Mechanical Engineering, University College Dublin, Belfield, Dublin 4, Ireland. E-mail: scott.rickard@ucd.ie

$¥$ Siemens Corporate Research, 755 College Road East, Princeton, NJ 08540, USA.

$\S$ Department of Electrical Engineering, Princeton University, Princeton, NJ 08544, USA. 
and the received signal is a summation of the reflections characterized by $\mathcal{L}(a, b)$, the wideband spreading function ${ }^{1}$,

$$
y(t)=\iint \mathcal{L}(a, b) \frac{1}{\sqrt{|a|}} x\left(\frac{t-b}{a}\right) \mathrm{d} a \mathrm{~d} b .
$$

We call a time-scale channel a wideband channel when the wideband spreading function has finite support. Due to the physical limitations of signal propagation, it is reasonable to expect that $\mathcal{L}(a, b)$ has finite support. The maximum possible rate of change in path length, which is constrained by the speeds of the objects in the environment, limits the support of $\mathcal{L}(a, b)$ to a narrow range around the $a=1$ line. Causality and the propagation loss associated with increasing path length effectively limit the support of $\mathcal{L}(a, b)$ to a finite range in the $b$ direction. The support in the $a$ direction causes a spreading in scale of the transmitted signal, and the support in the $b$ direction causes a spreading in time of the transmitted signal. Thus, channels described by (2) are often referred to as doubly spread channels.

Many signals and signaling environments satisfy the narrowband condition, an assumption under which the time dilations or contractions are modeled as Doppler shifts. Under this assumption, each received reflection of the signal is assumed to be of the form,

$$
x_{\tau, \theta}(t)=x(t-\tau) e^{j 2 \pi \theta t} .
$$

In the narrowband channel model, the received signal is a superposition of time delayed and frequency shifted copies of the input and the channel is characterized by the narrowband spreading function $S(\theta, \tau)$,

$$
y(t)=\iint S(\theta, \tau) x(t-\tau) e^{j 2 \pi \theta t} \mathrm{~d} \tau \mathrm{d} \theta,
$$

where $S(\theta, \tau)$ typically has finite support in $\theta$ and $\tau$ due to the physical limitations of the channel. The span of this spreading in time and frequency has proven to be a crucial parameter in communication systems $[1,2,3]$. Regardless of whether the support constraint is satisfied or not, (4) is a time-frequency description of a general time-varying linear system,

$$
y(t)=\int h(t, \tau) x(t-\tau) \mathrm{d} \tau .
$$

When $S(\theta, \tau)$ has no support constraint, the transmitted waveform and environment need not satisfy the narrowband condition.

Kailath's pioneering work in his 1959 Master's thesis [4] and the concomitant development of the rake channel model provided a mathematical framework for capturing the energy associated with multiple transmission paths between transmitter

\footnotetext{
${ }^{1}$ We will assume that all integrals are taken over $(-\infty, \infty)$ unless otherwise specified.
} 
and receiver using a discretization of the channel model. This work was furthered in 1963 by Bello, who proposed a discrete time-frequency characterization of the timevarying channel [2]. In [5], Sayeed and Aazhang reinterpreted this characterization from a diversity viewpoint, and used this canonical time-frequency channel characterization which combines a discrete set of time delayed and frequency shifted versions of the input signal,

$$
y(t)=\sum_{n=0}^{N} \sum_{k=-K}^{K} \hat{S}\left(\frac{k}{T}, \frac{n}{W}\right) x\left(t-\frac{n}{W}\right) e^{j 2 \pi k t / T},
$$

where

$$
\hat{S}(\theta, \tau):=\iint S\left(\theta^{\prime}, \tau^{\prime}\right) \operatorname{sinc}\left(\left(\tau-\tau^{\prime}\right) W\right) \operatorname{sinc}\left(\left(\theta-\theta^{\prime}\right) T\right) e^{-j \pi\left(\theta-\theta^{\prime}\right) T} \mathrm{~d} \theta^{\prime} \mathrm{d} \tau^{\prime}
$$

to define a delay-Doppler RAKE receiver, a two-dimensional extension of the classic rake receiver. The delay-Doppler rake takes advantage of the inherent added channel diversity associated with time-varying narrowband channels [5]. While the narrowband assumption is satisfied in many wireless communication signal environments, many wireless systems are wideband due to the higher data rates and multiaccess techniques [6]. Thus we may expect, in light of differences in the narrowband and wideband models, some advantages to the development of a canonical time-scale channel characterization in wideband communication scenarios. Motivated by this, [7, 8] used the channel in (2) to derive a time-scale canonical channel model

$$
y(t)=\sum_{m, n} \frac{c_{m, n}}{a_{0}^{m / 2}} x\left(\frac{t-n b_{0} a_{0}^{m}}{a_{0}^{m}}\right),
$$

where $a_{0}, b_{0}$ are related to channel and signal characteristics, and

$$
c_{m, n}=\iint \mathcal{L}(a, b) \operatorname{sinc}\left(m-\frac{\ln a}{\ln a_{0}}\right) \operatorname{sinc}\left(n-\frac{b}{a b_{0}}\right) \mathrm{d} a \mathrm{~d} b .
$$

An identical formula has been derived independently in [9, 10]. There is a difference, however, in the physical meaning of the decomposition in (8) between $[7,8]$ and $[9,10]$. We will discuss this difference in Section 4 where we will also present our point of view on canonical channel models. For us, a canonical model will refer to a time-varying linear system applied to a particular class of transmit signals whose output is measured through a particular observation procedure. For example, the time-frequency canonical model derived in [5] is based on bandlimited transmit signals observed at the receiver over a finite observation horizon (i.e., a time-domain limited receiver). As we discuss below, the time-scale canonical model can be derived from bandlimited transmit signals being observed at a scale-domain limited receiver. Furthermore, the new third canonical frequency-scale channel model introduced in this paper can be derived from scale-limited transmit signals being received at a time-domain limited receiver. We elaborate on this point in Section 4. 
Based on the above interpretation, in Section 4.1 we introduce a frequency-scale time-varying channel model of the form:

$$
y(t)=\int_{-\infty}^{\infty} \int_{0}^{\infty} \hat{\rho}(\omega, a) e^{j 2 \pi \omega t} \frac{1}{\sqrt{a}} x\left(\frac{t}{a}\right) \mathrm{d} a \mathrm{~d} \omega
$$

which is equivalent to (5) for positive time supported input signals and positive time horizon receivers, as we show in Appendix C. The canonical channel model derived from (10) is

$$
y(t)=\sum_{m, n} c_{m, n} e^{j 2 \pi m t /\left(T_{2}-T_{1}\right)} 1_{\left[T_{1}, T_{2}\right]}(t) a_{0}^{n / 2} x\left(a_{0}^{n} t\right)
$$

where

$$
1_{\left[T_{1}, T_{2}\right]}(t)=\left\{\begin{array}{lll}
1 & : & T_{1} \leq t \leq T_{2} \\
0 & : & \text { otherwise }
\end{array}\right.
$$

and $c_{m, n}$ are coefficients which depend on the span of the observation time horizon $\left(T_{2}-T_{1}\right)$, the scale domain bandwidth, and frequency-scale spreading function (see Equation (81)).

Each of the three doubly spread canonical channel models discussed above motivates the development of a different two-dimensional rake receiver. A delay-dilation rake receiver based on the canonical time-scale channel characterization $[9,11,10]$ leverages the diversity in wideband signaling environments in the same way that the delay-Doppler rake leverages the diversity in narrowband signaling environments [5]. Such a channel model and receiver may be particularly useful for ultra-wideband signaling due to the extremely wide transmission signal bandwidth $[12,13]$.

1.1. Outline of paper. In Section 2 we review background material on continuous narrowband (time-frequency) and wideband (time-scale) channel characterizations and examine simple one-path delay-Doppler and one-path delay-dilation channels in the framework of these representations. We derive and discuss the mapping between time-frequency and time-scale kernel operators and note that there exist timefrequency channels with no corresponding time-scale channel. In Section 3 we develop a general technique for the generation of canonical channel models and demonstrate the application of the technique to time-frequency and time-scale kernel operators. In Section 4 we discuss the interpretation and derivation of these canonical models from reasonable processing assumptions on the transmit and receive waveforms. In Section 4.1 we propose a frequency-scale canonical channel characterization based on the translation operators in frequency and scale. We conclude and propose future work in Section 5.

\section{Continuous Narrowband and Wideband Channel Characterizations.}

In this section we review and discuss the time-frequency and time-scale channel models and examine some simple channels to gain some intuition concerning the characterizations. The time-frequency description is a general time-varying linear system 
characterization. However, in a slight abuse of nomenclature, we will refer to all channel characterizations which can be related to the channel described by $S(\theta, \tau)$ via Fourier transforms and phase factors as narrowband channels. Specifically, in this section, we discuss twelve such equivalent characterizations which were first explored by Kailath [4], Zadeh [14], and Bello [2]. We call these "narrowband" characterizations because when $S(\theta, \tau)$ has finite support, the characterization is typically used only in narrowband systems and is not appropriate for wideband signals. We will only discuss the support condition constraint on $S(\theta, \tau)$ for the narrowband characterizations when relevant, and consider the more general case where there is no such constraint on the support of $S(\theta, \tau)$. Similarly, we will refer to channel characterizations based on the time-scale kernel $\mathcal{L}(a, b)$ as wideband characterizations because they are typically used in a wideband setting [15].

2.1. Narrowband Characterizations. In this section, we develop a general technique for the generation of canonical channel models and demonstrate the application of the technique to time-frequency and time-scale kernel operators.

The linear time-varying channel is characterized by the time-varying impulse response $h(t, \tau)$ which denotes the response of the channel at time $t$ to an impulse at time $t-\tau$. The channel input-output relationship is thus,

$$
y(t)=\int h(t, \tau) x(t-\tau) \mathrm{d} \tau .
$$

Such notation is used in, for example, $[16,17,18,19,5]$.

Another possible notation for the time-varying impulse response is

$$
y(t)=\int k_{0}(t, \tau) x(\tau) \mathrm{d} \tau
$$

with the interpretation that $k_{0}(t, \tau)$ is the response of the channel at time $t$ to an impulse at time $\tau$. This is the formulation used in, for example, [20, 21, 22]. Bello [2] calls $k_{0}(t, \tau)$ a kernel system function and notes the obvious correspondence between the two representations, $h(t, \tau)=k_{0}(t, t-\tau)$. Bello [2] defines four equivalent representations of the time-varying channel represented by $k_{0}(t, \tau)$ that map the time or frequency representations of the input into the time or frequency representations of the output. We define these four kernel functions,

$$
\begin{aligned}
y(t) & =\int k_{0}(t, \tau) x(\tau) \mathrm{d} \tau & Y(\theta) & =\int k_{1}(\theta, \tau) x(\tau) \mathrm{d} \tau \\
y(t) & =\int k_{2}(t, \nu) X(\nu) \mathrm{d} \nu & Y(\theta) & =\int k_{3}(\theta, \nu) X(\nu) \mathrm{d} \nu .
\end{aligned}
$$

The kernel system functions can be transformed into one another using the Fourier transform. For example, the kernel function that maps the input time domain to the output time domain $\left(k_{0}(t, \tau)\right)$ and the kernel function that maps the input time domain to the output frequency domain $\left(k_{1}(\theta, \tau)\right)$ are Fourier transforms of one another 
with respect to the first argument. We can summarize the relationships among the kernel system functions as follows,

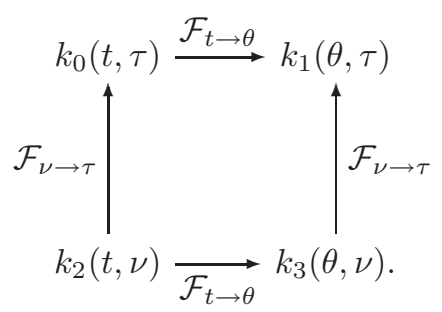

That is,

$$
\begin{array}{ll}
k_{0}(t, \tau)=\int k_{2}(t, \nu) e^{-j 2 \pi \nu \tau} \mathrm{d} \nu & k_{1}(\theta, \tau)=\int k_{0}(t, \tau) e^{-j 2 \pi t \theta} \mathrm{d} t \\
k_{2}(t, \nu)=\int k_{3}(\theta, \nu) e^{j 2 \pi \theta t} \mathrm{~d} \theta & k_{3}(\theta, \nu)=\int k_{1}(\theta, \tau) e^{j 2 \pi \tau \nu} \mathrm{d} \tau .
\end{array}
$$

The direction of the Fourier transform between $k_{0}$ and $k_{2}$ (and also between $k_{1}$ and $k_{3}$ ) is opposite to convention; We take the Fourier transform with respect to a "frequency" variable $(\nu)$ and replace it with a "time" variable $(\tau)$. This is necessary to be consistent with the kernel functions as defined in (15).

Bello [2] provides the following useful interpretation of the kernel system functions,

- The response to input $\delta\left(t-t_{0}\right)$ is time function $k_{0}\left(t, t_{0}\right)$ with spectrum $k_{1}\left(\theta, t_{0}\right)$,

- The response to input $e^{j 2 \pi \theta_{0} t}$ is time function $k_{2}\left(t, \theta_{0}\right)$ with spectrum $k_{3}(\theta$, $\left.\theta_{0}\right)$,

and also notes, by simple inspection of (15), that $k_{0}$ and $k_{3}$ are time-frequency duals of one another, as are $k_{1}$ and $k_{2}$.

Despite the simple input-output interpretations, the kernel system functions often lack intuitive physical interpretations [4]. For this reason, Bello [2] and Kailath [23] examined eight other system function characterizing the linear time-varying channel. These eight system functions are (13); its time-frequency dual,

$$
Y(\theta)=\int G(\theta, \nu) X(\theta-\nu) \mathrm{d} \nu
$$

the three functions obtained by taking the Fourier transform of $h(t, \tau)$ with respect to $t, \tau$, and both $t$ and $\tau$; and the three functions obtained by taking the inverse Fourier transform of $G(\theta, \nu)$ with respect to $\theta, \nu$, and both $\theta$ and $\nu$. These eight functions are listed in Table 1.

In the current literature, $h(t, \tau)$ is usually referred to as the time-varying impulse response, (e.g., $[16,17,18,19,5])$ and the delay-Doppler spreading function, $S(\theta, \tau)$, is known simply as the spreading function (e.g., [16, 17, 18, 19, 5, 21]). Unfortunately, $k_{0}(t, \tau)$ is also commonly referred to as the time-varying impulse response (e.g., [20, 
TABLE 1

Eight system functions characterizing the linear time-varying channel, their function names from Bello [2], and their associated input-output relationship or definition.

\begin{tabular}{|c|c|c|}
\hline$h(t, \tau)$ & input delay spread function & $y(t)=\int h(t, \tau) x(t-\tau) \mathrm{d} \tau$ \\
\hline$S(\theta, \tau)$ & delay-Doppler spreading function & $S(\theta, \tau)=\int h(t, \tau) e^{-j 2 \pi t \theta} \mathrm{d} t$ \\
\hline$T(t, \nu)$ & time-varying transfer function & $T(t, \nu)=\int h(t, \tau) e^{-j 2 \pi \tau \nu} \mathrm{d} \tau$ \\
\hline$H(\theta, \nu)$ & output Doppler spread function & $H(\theta, \nu)=\iint h(t, \tau) e^{-j 2 \pi(t \theta+\tau \nu)} \mathrm{d} t \mathrm{~d} \tau$ \\
\hline$G(\theta, \nu)$ & input Doppler spread function & $Y(\theta)=\int G(\theta, \nu) X(\theta-\nu) \mathrm{d} \nu$ \\
\hline$V(t, \nu)$ & Doppler-delay spreading function & $V(t, \nu)=\int G(\theta, \nu) e^{j 2 \pi \theta t} \mathrm{~d} \theta$ \\
\hline$M(\theta, \tau)$ & $\begin{array}{l}\text { frequency dependent } \\
\text { modulation function }\end{array}$ & $M(\theta, \tau)=\int G(\theta, \nu) e^{j 2 \pi \nu \tau} \mathrm{d} \nu$ \\
\hline$g(t, \tau)$ & output delay spread function & $g(t, \tau)=\iint G(\theta, \nu) e^{j 2 \pi(\theta t+\nu \tau)} \mathrm{d} \theta \mathrm{d} \nu$ \\
\hline
\end{tabular}

21]). We will refer to $k_{0}(t, \tau)$ as the time-varying impulse response kernel to avoid confusion.

The relationships among the eight functions via duality and the Fourier transform are summarized in the following diagram. Duality is represented by a dotted line.

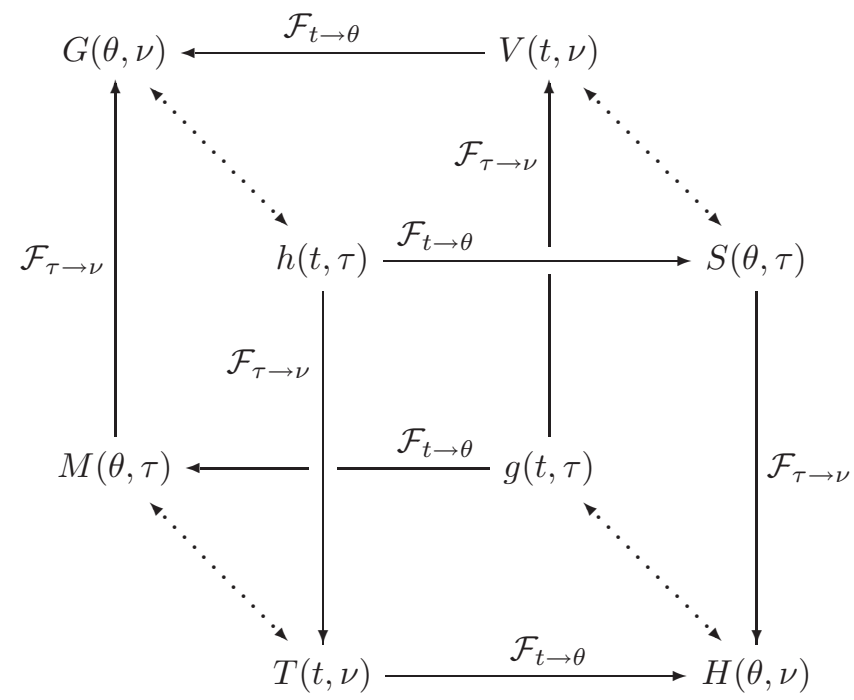

We can derive the following input-output relationships,

$$
\begin{array}{ll}
y(t)=\int h(t, \tau) x(t-\tau) \mathrm{d} \tau & y(t)=\iint S(\theta, \tau) e^{j 2 \pi \theta t} x(t-\tau) \mathrm{d} \theta \mathrm{d} \tau \\
y(t)=\int T(t, \nu) e^{j 2 \pi \nu t} X(\nu) \mathrm{d} \nu & Y(\theta)=\int H(\theta-\nu, \nu) X(\nu) \mathrm{d} \nu
\end{array}
$$


and

$$
\begin{array}{rlrl}
Y(\theta) & =\int G(\theta, \nu) X(\theta-\nu) \mathrm{d} \nu & Y(\theta) & =\iint V(t, \nu) e^{-j 2 \pi t \theta} X(\theta-\nu) \mathrm{d} t \mathrm{~d} \nu \\
Y(\theta) & =\int M(\theta, \tau) e^{-j 2 \pi \tau \theta} x(\tau) \mathrm{d} \tau & y(t)=\int g(t-\tau, \tau) x(\tau) \mathrm{d} \tau .
\end{array}
$$

We can relate the eight system functions to the four kernel system functions as follows,

$$
\begin{aligned}
k_{0}(t, \tau) & = & h(t, t-\tau) &
\end{aligned}
$$

$S(\theta, \tau)$ and $V(t, \nu)$ are distinctive in that their input-output characterizations and relations to the kernel system functions involve double integrals. In fact, it is the double integral formulation involving $S(\theta, \tau)$ in (20) with the interpretation that the output is a superposition of time-delayed and Doppler-shifted copies of the input that makes $S(\theta, \tau)$ an extremely useful characterization. For completeness, we note the inverse relations,

$$
\begin{aligned}
S(\theta, \tau) & =\iint k_{1}(\nu, t) e^{j 2 \pi(t+\tau)(\nu-\theta)} \mathrm{d} \nu \mathrm{d} t \\
V(t, \nu) & =\iint k_{2}(\tau, \theta) e^{j 2 \pi(t-\tau)(\theta+\nu)} \mathrm{d} \tau \mathrm{d} \theta
\end{aligned}
$$

and note the following relationship between the dual characterizations $h(t, \tau)$ and $G(\theta, \nu)$,

$$
\begin{aligned}
h(t, \tau) & =\iint G(\theta, \nu) e^{j 2 \pi \theta t} e^{-j 2 \pi(t-\tau)(\theta-\nu)} \mathrm{d} \theta \mathrm{d} \nu \\
G(\theta, \nu) & =\iint h(t, \tau) e^{-j 2 \pi t \theta} e^{j 2 \pi(\theta-\nu)(t-\tau)} \mathrm{d} t \mathrm{~d} \tau .
\end{aligned}
$$

Although less commonly used in the literature, $k_{3}(\theta, \nu)$ plays a pivotal role in understanding the narrowband and wideband characterizations [7]. We note the mapping between $k_{3}$ and $S$,

$$
\begin{aligned}
k_{3}(\theta, \nu) & =\int S(\theta-\nu, \tau) e^{-j 2 \pi \tau \nu} \mathrm{d} \tau \\
S(\theta, \tau) & =\int k_{3}(\theta+\nu, \nu) e^{j 2 \pi \tau \nu} \mathrm{d} \nu,
\end{aligned}
$$

which can be derived directly from the input-output channel characterizations. In the kernel system formulation of the channel, the outputs could be simply expressed in term of the kernel functions for inputs that were impulses in time and frequency. For the above characterizations, these relations are: 
- The response to $\delta\left(t-t_{0}\right)$ is $h\left(t, t-t_{0}\right)$ with spectrum $M\left(\theta, t_{0}\right) e^{-j 2 \pi \theta t_{0}}$.

- The response to $e^{j 2 \pi \theta_{0} t}$ is $T\left(t, \theta_{0}\right) e^{j 2 \pi t \theta_{0}}$ with spectrum $H\left(\theta-\theta_{0}, \theta_{0}\right)$.

For clarity, we display just the front face of the cube in (19), which details the Fourier transform relationships among the four most commonly used system functions.

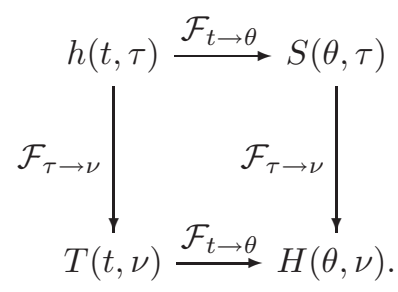

In order to get some intuition concerning the channel characterization functions (both the four kernel functions (15) and the additional eight characterizations listed in Table 1, we examine simple channels. Consider the time-invariant channel that consists of a pure delay.

$$
x(t) \rightarrow \text { channel } \rightarrow x\left(t-\tau_{0}\right)
$$

In the case of the time-varying impulse response kernel, this channel is represented by $k_{0}(t, \tau)=\delta\left(t-\tau-\tau_{0}\right)$. In the case of the time-varying impulse response, this channel is represented by $h(t, \tau)=\delta\left(\tau-\tau_{0}\right)$. Plots of these two functions are displayed in Figure 1. One useful attribute of a system function is for a visual inspection of the function to readily reveal some physical properties of the channel. In the case of Figure 1, we see that, for $k_{0}(t, \tau)$, a diagonal delta function line crossing through $\left(0,-\tau_{0}\right)$ and $\left(\tau_{0}, 0\right)$ arises from a delay of $\tau_{0}$. For $h(t, \tau)$, a delay of $\tau_{0}$ corresponds to a horizontal delta function line $\tau_{0}$ from the origin. A channel with several reflections (i.e., several different delays), would thus correspond to a system function with several parallel delta function lines. When the channel involves both a simple delay and a Doppler shift, the simple delta function lines for both $k_{0}(t, \tau)$ and $h(t, \tau)$ are modulated by the Doppler shift. Table 2 displays the twelve system functions for the delay and delay-Doppler channels. The system function with the simplest form is $S(\theta, \tau)$ which is the product of delta functions. From this, we interpret a region of localized energy in $S(\theta, \tau)$ centered at $\left(\theta_{0}, \tau_{0}\right)$ as arising from an echo path with delay $\tau_{0}$ and Doppler shift $\theta_{0}$; see Figure 2 .

2.2. Wideband Characterizations. Starting from the wideband channel characterization,

$$
y(t)=\iint \mathcal{L}(a, b) \frac{1}{\sqrt{|a|}} x\left(\frac{t-b}{a}\right) \mathrm{d} a \mathrm{~d} b
$$




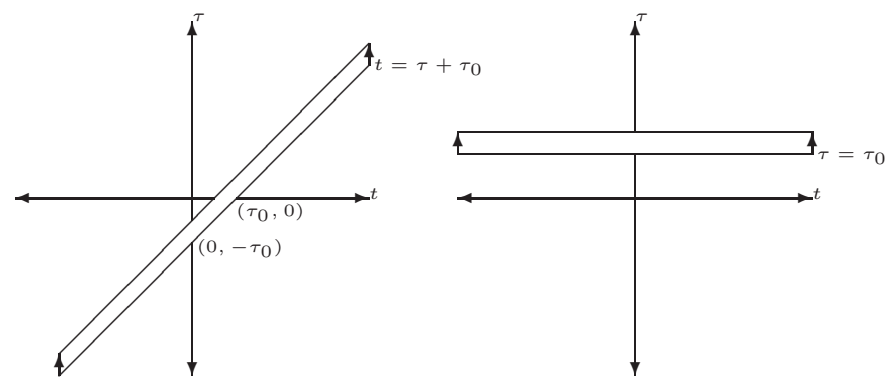

FIG. 1. $k_{0}(t, \tau)$ (left) and $h(t, \tau)$ (right) for the delay-by- $\tau_{0}$ channel.

TABLE 2

Time-frequency characterization functions for the one-path delay and one-path delay-Doppler channels. $S(\theta, \tau)$ has a very simple form for the one-path delay-Doppler channel.

\begin{tabular}{|c|c|c|}
\cline { 2 - 3 } \multicolumn{1}{c|}{} & $y(t)=x\left(t-\tau_{0}\right)$ & $y(t)=x\left(t-\tau_{0}\right) e^{j 2 \pi \theta_{0} t}$ \\
\hline$k_{0}(t, \tau)$ & $\delta\left(t-\tau-\tau_{0}\right)$ & $\delta\left(t-\tau-\tau_{0}\right) e^{j 2 \pi \theta_{0} t}$ \\
\hline$k_{1}(\theta, \tau)$ & $e^{-j 2 \pi\left(\tau+\tau_{0}\right) \theta}$ & $e^{-j 2 \pi\left(\tau+\tau_{0}\right)\left(\theta-\theta_{0}\right)}$ \\
\hline$k_{2}(t, \nu)$ & $e^{j 2 \pi\left(t-\tau_{0}\right) \nu}$ & $e^{j 2 \pi t\left(\nu+\theta_{0}\right)} e^{-j 2 \pi \tau_{0} \nu}$ \\
\hline$k_{3}(\theta, \nu)$ & $\delta(\theta-\nu) e^{-j 2 \pi \tau_{0} \nu}$ & $\delta\left(\theta-\nu-\theta_{0}\right) e^{-j 2 \pi \tau_{0} \nu}$ \\
\hline \hline$h(t, \tau)$ & $\delta\left(\tau-\tau_{0}\right)$ & $\delta\left(\tau-\tau_{0}\right) e^{j 2 \pi t \theta_{0}}$ \\
\hline$S(\theta, \tau)$ & $\delta\left(\tau-\tau_{0}\right) \delta(\theta)$ & $\delta\left(\tau-\tau_{0}\right) \delta\left(\boldsymbol{\theta}-\boldsymbol{\theta}_{0}\right)$ \\
\hline$T(t, \nu)$ & $e^{-j 2 \pi \tau_{0} \nu}$ & $e^{j 2 \pi \theta_{0} t} e^{-j 2 \pi \tau_{0} \nu}$ \\
\hline$H(\theta, \nu)$ & $e^{-j 2 \pi \tau_{0} \nu} \delta(\theta)$ & $e^{-j 2 \pi \tau_{0} \nu} \delta\left(\theta-\theta_{0}\right)$ \\
\hline$G(\theta, \nu)$ & $e^{-j 2 \pi \tau_{0} \theta} \delta(\nu)$ & $e^{-j 2 \pi \tau_{0}\left(\theta-\theta_{0}\right)} \delta\left(\nu-\theta_{0}\right)$ \\
\hline$V(t, \nu)$ & $\delta\left(t-\tau_{0}\right) \delta(\nu)$ & $e^{j 2 \pi \tau_{0} \theta_{0}} \delta\left(t-\tau_{0}\right) \delta\left(\nu-\theta_{0}\right)$ \\
\hline$M(\theta, \tau)$ & $e^{-j 2 \pi \tau_{0} \theta}$ & $e^{-j 2 \pi \tau_{0}\left(\theta-\theta_{0}\right)} e^{j 2 \pi \tau \theta_{0}}$ \\
\hline$g(t, \tau)$ & $\delta\left(t-\tau_{0}\right)$ & $e^{j 2 \pi \tau_{0} \theta_{0}} \delta\left(t-\tau_{0}\right) e^{j 2 \pi \tau \theta_{0}}$ \\
\hline
\end{tabular}
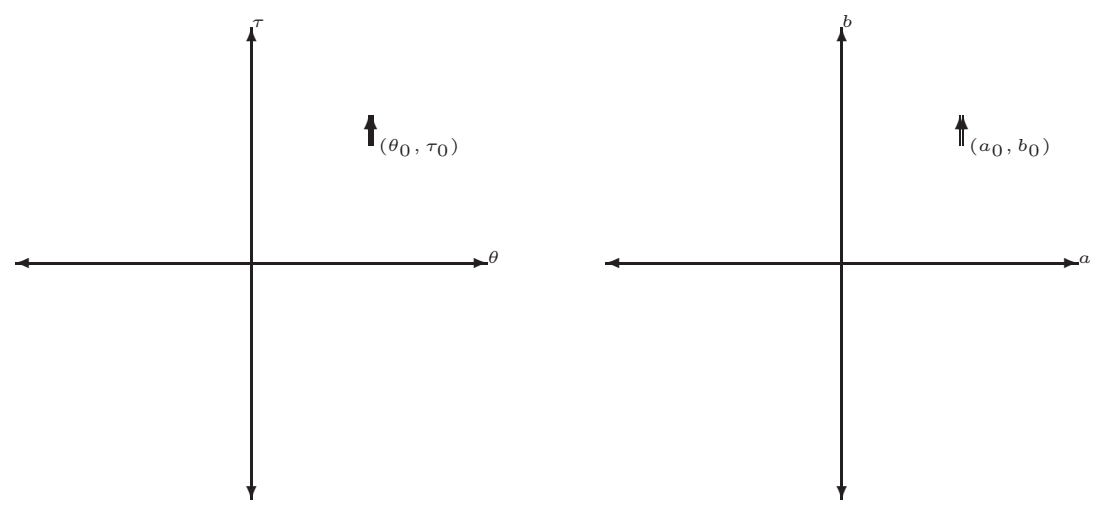

FIG. 2. $S(\theta, \tau)$ for one-path channel with delay $\tau_{0}$ and Doppler shift $\theta_{0}$ (left); $\mathcal{L}(a, b)$ for one-path channel with delay $b_{0}$ and time dilation $a_{0}$ (right). 
TABLE 3

Time-scale characterization functions for the one-path delay and one-path delay-dilation channels.

\begin{tabular}{|c|c|c|}
\cline { 2 - 3 } \multicolumn{1}{c|}{} & $y(t)=x\left(t-b_{0}\right)$ & $y(t)=\frac{1}{\sqrt{\left|a_{0}\right|}} x\left(\frac{t-b_{0}}{a_{0}}\right)$ \\
\hline $\mathcal{L}(a, b)$ & $\delta(a-1) \delta\left(b-b_{0}\right)$ & $\delta\left(a-a_{0}\right) \delta\left(b-b_{0}\right)$ \\
\hline $\mathcal{L}^{(2)}(a, \theta)$ & $\delta(a-1) e^{-j 2 \pi b_{0} \theta}$ & $\delta\left(a-a_{0}\right) e^{-j 2 \pi b_{0} \theta}$ \\
\hline
\end{tabular}

we derive the frequency domain to frequency domain mapping,

$$
\begin{aligned}
Y(\theta) & =\iiint \mathcal{L}(a, b) \frac{1}{\sqrt{|a|}} x\left(\frac{t-b}{a}\right) e^{-j 2 \pi t \theta} \mathrm{d} a \mathrm{~d} b \mathrm{~d} t \\
& =\iiint \mathcal{L}(a, b) \sqrt{|a|} x\left(t^{\prime}\right) e^{-j 2 \pi\left(a t^{\prime}+b\right) \theta} \mathrm{d} a \mathrm{~d} b \mathrm{~d} t^{\prime} \\
& =\iint \mathcal{L}(a, b) \sqrt{|a|} X(a \theta) e^{-j 2 \pi b \theta} \mathrm{d} a \mathrm{~d} b
\end{aligned}
$$

and defining,

$$
\mathcal{L}^{(2)}(a, \theta)=\int \mathcal{L}(a, b) e^{-j 2 \pi b \theta} \mathrm{d} b
$$

we obtain

$$
Y(\theta)=\int \mathcal{L}^{(2)}(a, \theta) \sqrt{|a|} X(a \theta) \mathrm{d} a .
$$

Table 3 displays the wideband characterization functions for the one-path delay and one-path delay-dilation channels. In the narrowband case, $S(\theta, \tau)$ is the product of delta functions for the one-path delay-Doppler channel; In the wideband case, the one-path delay-dilation channel is the product of delta functions. We interpret a region of concentrated energy in $\mathcal{L}(a, b)$ centered at $\left(a_{0}, b_{0}\right)$ as arising from an echo path with delay $b_{0}$ and dilation parameter $a_{0}$.

2.3. Narrowband and Wideband Correspondence. In this section we briefly examine the correspondence between the narrowband and wideband channel models. More specifically, we wish to link the narrowband channel model characterized by the dozen system functions discussed above, one of which was described by the time-frequency integral operator,

$$
\mathcal{N}_{S} x(t):=\iint S(\theta, \tau) x(t-\tau) e^{j 2 \pi \theta t} \mathrm{~d} \tau \mathrm{d} \theta
$$

to the wideband channel description embodied in the time-scale integral operator,

$$
\mathcal{W}_{\mathcal{L}} x(t):=\iint \mathcal{L}(a, b) \frac{1}{\sqrt{|a|}} x\left(\frac{t-b}{a}\right) \mathrm{d} a \mathrm{~d} b .
$$


We are interested in the mapping between $S$ and $\mathcal{L}$ for $\mathcal{N}_{S}=\mathcal{W}_{\mathcal{L}}$. The approach taken here differs from the traditional interpretation of the narrowband characterization as an approximation of the wideband characterization when applied to narrowband signals. This approximation is discussed in detail in, for example, $[24,25,15,26,27$, $28,29]$. We do not consider the narrowband description of the channel as an approximation of the wideband channel, but rather look at the two descriptions without constraining the properties of the input signal.

We first establish the relation from wideband to narrowband, showing that for every time-scale kernel, there exists a corresponding time-frequency kernel. Starting from (38), we have

$$
\begin{aligned}
y(t) & =\iint \mathcal{L}(a, b) \frac{1}{\sqrt{|a|}} x\left(\frac{t-b}{a}\right) \mathrm{d} a \mathrm{~d} b \\
& =\int\left(\int \sqrt{|a|} \mathcal{L}(a, t-a \tau) \mathrm{d} a\right) x(\tau) \mathrm{d} \tau,
\end{aligned}
$$

and therefore,

$$
k_{0}(t, \tau)=\int \sqrt{|a|} \mathcal{L}(a, t-a \tau) \mathrm{d} a
$$

Returning to the mapping from $\mathcal{L}(a, b)$ to the narrowband characterizations, starting from (40), the remaining system functions can be related to $\mathcal{L}(a, b)$ as follows,

$$
h(t, \tau)=\int \sqrt{|a|} \mathcal{L}(a,(1-a) t+a \tau) \mathrm{d} a
$$

and, taking the Fourier transform of (41) with respect to $t$, we obtain,

$$
S(\theta, \tau)=\iint \sqrt{|a|} \mathcal{L}(a,(1-a) t+a \tau) e^{-j 2 \pi \theta t} \mathrm{~d} a \mathrm{~d} t .
$$

Using (40), it is possible to relate $\mathcal{L}$ to all twelve narrowband representations [7].

It is also possible to express $\mathcal{L}(a, b)$ in terms of $S(\theta, \tau)$,

$$
\mathcal{L}(a, b)=\iint \frac{|\theta|}{\sqrt{|a|}} S((1-a) \theta, \tau) e^{j 2 \pi \theta(b-a \tau)} \mathrm{d} \theta \mathrm{d} \tau
$$

although the mapping relies on the assumption that the input signal has no DC component; see [7] for a discussion of this mapping. We can observe from (36) that, in the wideband model, the DC input component can only affect the DC output component. Intuitively, it is clear that rescaling the time axis and shifting in time a DC signal does not have any effect, and all the time-scale channel can do is amplify or attenuate the DC component of a signal. This is not the case in the narrowband model. For example, from $k_{3}(\theta, \nu)$ in (15) it is clear that the DC input signal component can affect any output frequency component. Therefore, there are time-frequency characterizations which have no corresponding time-scale representation. 
We look to some simple channel models and examine the mappings between $\mathcal{L}$ and $S$. We first consider the wideband (delay-dilation) single path channel,

$$
\mathcal{L}(a, b)=\delta\left(a-a_{0}\right) \delta\left(b-b_{0}\right)
$$

It follows from (42) that,

$$
S(\theta, \tau)=\left\{\begin{array}{cc}
\frac{\sqrt{\left|a_{0}\right|}}{\left|1-a_{0}\right|} e^{-j 2 \pi \theta \frac{b_{0}-a_{0} \tau}{1-a_{0}}} \quad: \quad a_{0} \neq 1 \\
\delta(\theta) \delta\left(\tau-b_{0}\right) & : \quad a_{0}=1
\end{array}\right.
$$

and, substituting this into (4) we obtain $y(t)=x_{a_{0}, b_{0}}(t)$, as expected.

We can derive the time-varying impulse response characterization $h(t, \tau)$ for the wideband single path channel,

$$
\begin{aligned}
h(t, \tau) & =\int S(\theta, \tau) e^{j 2 \pi \theta t} \mathrm{~d} \theta \\
& =\int \frac{\sqrt{\left|a_{0}\right|}}{\left|1-a_{0}\right|} e^{-j 2 \pi \theta \frac{b_{0}-a_{0} \tau}{1-a_{0}}} e^{j 2 \pi \theta t} \mathrm{~d} \theta \\
& =\frac{\sqrt{\left|a_{0}\right|}}{\left|1-a_{0}\right|} \delta\left(\frac{b_{0}-a_{0} \tau-\left(1-a_{0}\right) t}{1-a_{0}}\right) \\
& =\sqrt{\left|a_{0}\right|} \delta\left(b_{0}-a_{0} \tau-\left(1-a_{0}\right) t\right)
\end{aligned}
$$

which is also valid when $a_{0}=1$. We can compare this result to that of the single narrowband path (delay by $\tau_{0}$, Doppler shift by $\theta_{0}$ ) channel, $h(t, \tau)=\delta\left(\tau-\tau_{0}\right) e^{j 2 \pi t \theta_{0}}$. The wideband path gives rise to a delta function line with slope $\frac{a_{0}-1}{a_{0}}$ intersecting the $\tau$-axis at $b_{0} / a_{0}$; The narrowband path gives rise to a modulated delta function line parallel to the $t$-axis intersecting the $\tau$-axis at $\tau_{0}$.

We now turn to the expression of the narrowband (delay-Doppler) single path in the wideband model:

$$
S(\theta, \tau)=\delta\left(\theta-\theta_{0}\right) \delta\left(\tau-\tau_{0}\right)
$$

If we ignore the difficulties arising from the instabilities on the $a=1$ line [7], it follows from (43) that

$$
\mathcal{L}(a, b)=\frac{\left|\theta_{0}\right|}{\sqrt{|a|}(1-a)^{2}} e^{j 2 \pi \theta_{0} \frac{b-a \tau_{0}}{1-a}}
$$

and, substituting this into (2), we indeed obtain, $y(t)=x_{\tau_{0}, \theta_{0}}(t)$.

The various channel characterizations for the simple one-path models (including the time-invariant one-path model) are displayed in Table 4 . We note that the onepath delay-dilation channel requires infinite support in time-frequency (45) whereas it requires only point support in time-scale (44). On the other hand, the one-path delay-Doppler channel requires infinite support in time-scale (48) whereas it requires 
TABLE 4

Time-frequency and time-scale characterizations for the one-path delay-Doppler and one-path delay-dilation channels.

\begin{tabular}{|l|l|l|l|}
\cline { 2 - 4 } \multicolumn{1}{c|}{} & one-path delay only & $\begin{array}{c}\text { one-path delay-Doppler } \\
\theta_{0} \neq 0\end{array}$ & $\begin{array}{l}\text { one-path delay-dilation } \\
a_{0} \neq 1\end{array}$ \\
\hline$S(\theta, \tau)$ & $\delta(\theta) \delta\left(\tau-t_{0}\right)$ & $\delta\left(\boldsymbol{\theta}-\boldsymbol{\theta}_{\mathbf{0}}\right) \boldsymbol{\delta}\left(\boldsymbol{\tau}-\tau_{\mathbf{0}}\right)$ & $\frac{\sqrt{\left|a_{0}\right|}}{\left|1-a_{0}\right|} e^{-j 2 \pi \theta \frac{b_{0}-a_{0} \tau}{1-a_{0}}}$ \\
\hline $\mathcal{L}(a, b)$ & $\delta(a-1) \delta\left(b-t_{0}\right)$ & $\frac{\left|\theta_{0}\right|}{\sqrt{|a|}(1-a)^{2}} e^{j 2 \pi \theta_{0} \frac{b-a \tau_{0}}{1-a}}$ & $\boldsymbol{\delta}\left(\boldsymbol{a}-\boldsymbol{a}_{\mathbf{0}}\right) \boldsymbol{\delta}\left(\boldsymbol{b}-\boldsymbol{b}_{\mathbf{0}}\right)$ \\
\hline$h(t, \tau)$ & $\delta\left(\tau-t_{0}\right)$ & $\delta\left(\tau-\tau_{0}\right) e^{j 2 \pi t \theta_{0}}$ & $\sqrt{\left|a_{0}\right|} \delta\left(\left(1-a_{0}\right) t+a_{0} \tau-b_{0}\right)$ \\
\hline$k_{3}(\theta, \nu)$ & $\delta(\theta-\nu) e^{-j 2 \pi t_{0} \nu}$ & $\delta\left(\theta-\nu-\theta_{0}\right) e^{-j 2 \pi \tau_{0} \nu}$ & $\sqrt{\left|a_{0}\right|} e^{-j 2 \pi \theta b_{0}} \delta\left(\nu-a_{0} \theta\right)$ \\
\hline
\end{tabular}

only point support in time-frequency. Thus, since we are interested in channels which have finite support in time-frequency or time-scale (as we will see in the next sections), the choice of channel model is crucial and must be appropriate to the signaling environment (i.e., narrowband or wideband). Examination of $k_{3}(\theta, \nu)$ for the onepath channels reveals that it is possible (up to a scaling constant) for the one-path delay-Doppler and the one-path delay-dilation channels to have the same effect on a narrowband signal $\left(\mathrm{eg}, X(\nu)=\delta\left(\nu-\nu_{0}\right)\right)$ by setting $\tau_{0}=b_{0} / a_{0}$ and $\theta_{0}=v_{0}\left(\frac{a_{0}-1}{a_{0}}\right)$.

3. Discrete Canonical Channel Models. In this section we develop a general technique for the generation of canonical channel models and demonstrate the application of the technique to time-frequency and time-scale kernel operators.

3.1. The canonical rake receiver model. We begin with the derivation of the canonical model associated with the standard rake receiver. The classic expression of the sampling theorem for a signal $X(\nu)$ with support $(-W / 2, W / 2)$ is

$$
x(t)=\sum_{n=-\infty}^{\infty} x\left(\frac{n}{W}\right) \frac{\sin \left(\pi W\left(t-\frac{n}{W}\right)\right)}{\pi W\left(t-\frac{n}{W}\right)} .
$$

An alternative formulation of the sampling theorem [19] is obtained by defining $g(t)=$ $x(\alpha-t)$,

$$
g(t)=\sum_{n=-\infty}^{\infty} g\left(\frac{n}{W}\right) \frac{\sin \left(\pi W\left(t-\frac{n}{W}\right)\right)}{\pi W\left(t-\frac{n}{W}\right)}
$$

and thus,

$$
x(\alpha-t)=\sum_{n=-\infty}^{\infty} x\left(\alpha-\frac{n}{W}\right) \frac{\sin \left(\pi W\left(t-\frac{n}{W}\right)\right)}{\pi W\left(t-\frac{n}{W}\right)} .
$$

Mapping $(\alpha, t) \rightarrow(t, \tau)$, we obtain,

$$
x(t-\tau)=\sum_{n=-\infty}^{\infty} x\left(t-\frac{n}{W}\right) \frac{\sin \left(\pi W\left(\tau-\frac{n}{W}\right)\right)}{\pi W\left(\tau-\frac{n}{W}\right)} .
$$


Following [19], substituting (52) into the time-varying impulse response channel characterization (13), we obtain

$$
\begin{aligned}
y(t) & =\int h(t, \tau) x(t-\tau) \mathrm{d} \tau \\
& =\sum_{n=-\infty}^{\infty} x\left(t-\frac{n}{W}\right) \underbrace{\left[\int h(t, \tau) \frac{\sin \left(\pi W\left(\tau-\frac{n}{W}\right)\right)}{\pi W\left(\tau-\frac{n}{W}\right)} \mathrm{d} \tau\right]}_{=h_{n}(t)} \\
& \approx \sum_{n=0}^{L:=\left\lceil T_{m} / W\right\rceil} x\left(t-\frac{n}{W}\right) h_{n}(t)
\end{aligned}
$$

where the approximation is made based on the assumption that the channel is causal and has finite multipath spread, $T_{m}$. That is, $h(t, \tau)=0, \forall \tau<0, \tau>T_{m}$. Under this assumption, the approximation (53c) corresponds to $h_{n}(t)$ for which the mainlobe of the sinc function overlaps with the support of the time-varying impulse response. The tapped-delay line in (53c) forms the basis for the classic rake receiver, where $h_{n}(t)$ 's are usually assumed to be independent of each other.

3.2. The canonical time-frequency model. We now proceed to examine the time-frequency canonical channel model which was originally derived in [5]. Alternative, but similar models are explored in $[30,31,32]$. The path we take in this derivation is essentially the same as that in [5]. We look at only the $(0, T)$ portion of the received waveform, that is, $y(t) 1_{(0, T)}(t)$. Starting from $(53 \mathrm{~b})$, we impose the $(0, T)$ restriction and obtain

$$
y(t) 1_{(0, T)}(t)=\sum_{n=-\infty}^{\infty} x\left(t-\frac{n}{W}\right)\left[\int h(t, \tau) 1_{(0, T)}(t) \operatorname{sinc}\left(W\left(\tau-\frac{n}{W}\right)\right) \mathrm{d} \tau\right] .
$$

Now we expand the $h(t, \tau) 1_{(0, T)}(t)$ term as a Fourier series,

$$
\begin{aligned}
h(t, \tau) 1_{(0, T)}(t) & =\sum_{k=-\infty}^{\infty} \frac{1}{T}\left[\int_{0}^{T} h\left(t^{\prime}, \tau\right) e^{-j 2 \pi k t^{\prime} / T} \mathrm{~d} t^{\prime}\right] e^{j 2 \pi k t / T} \\
& =\sum_{k=-\infty}^{\infty} \frac{1}{T} \underbrace{\left[\int_{-\infty}^{\infty} h\left(t^{\prime}, \tau\right) 1_{(0, T)}\left(t^{\prime}\right) e^{-j 2 \pi k t^{\prime} / T} \mathrm{~d} t^{\prime}\right]}_{\int_{-\infty}^{\infty} S(\theta, \tau) T \operatorname{sinc}\left(\left(\frac{k}{T}-\theta\right) T\right) e^{-j \pi(k-T \theta)} \mathrm{d} \theta} e^{j 2 \pi k t / T}
\end{aligned}
$$

which is valid for $t \in(0, T)$.

Substituting (55b) into (54) we obtain,

$$
y(t)=\sum_{n=-\infty}^{\infty} \sum_{k=-\infty}^{\infty} x\left(t-\frac{n}{W}\right) e^{j 2 \pi k t / T} \hat{S}\left(\frac{k}{T}, \frac{n}{W}\right)
$$

where,

$$
\hat{S}(\theta, \tau):=\iint S\left(\theta^{\prime}, \tau^{\prime}\right) \operatorname{sinc}\left(\left(\tau-\tau^{\prime}\right) W\right) \operatorname{sinc}\left(\left(\theta-\theta^{\prime}\right) T\right) e^{-j \pi\left(\theta-\theta^{\prime}\right) T} \mathrm{~d} \theta^{\prime} \mathrm{d} \tau^{\prime}
$$


(56) is valid for that part of any bandlimited signal received during $(0, T)$.

Under the path scatterer interpretation we assume that the channel introduces a maximum delay spread of $T_{m}$ and maximum Doppler spread of $B_{d}$, that is, $S(\theta, \tau)$ has support in $\left(-B_{d}, B_{d}\right) \times\left(0, T_{m}\right)$. In the smoothed version of $S(\theta, \tau)$ in $(57)$, if we consider only the terms in (56) where the main lobe of the smoothing kernel (which has size $(-1 / T, 1 / T)$-by- $(-1 / W, 1 / W))$ overlaps with the support of $S(\theta, \tau)$, we need only sum over $n=0, \ldots, N$ where $N=\left\lceil W T_{m}\right\rceil$ and $k=-K, \ldots, K$ where $K=\left\lceil T B_{d}\right\rceil$. We thus obtain the canonical representation of the time-frequency channel model,

$$
y(t)=\sum_{n=0}^{\left\lceil W T_{m}\right\rceil} \sum_{k=-\left\lceil T B_{d}\right\rceil}^{\left\lceil T B_{d}\right\rceil} x\left(t-\frac{n}{W}\right) e^{j 2 \pi k t / T} \hat{S}\left(\frac{k}{T}, \frac{n}{W}\right) .
$$

3.3. Restatement. The double sum time-frequency channel formulation (56) was obtained by assuming,

- the input signal is bandpass with bandwidth $W$, and

- the output signal is analyzed only for $t \in(0, T)$.

With these assumptions in mind, we define the following two projection operators,

$$
P_{T} x(t):=1_{[0, T]}(t) x(t)
$$

and,

$$
Q_{W} x(t):=\mathcal{F}^{-1}\left\{1_{[-W / 2, W / 2]}(\omega) \mathcal{F}\{x(t)\}(\omega)\right\},
$$

and using the following two operators, the translation operator,

$$
T_{\tau} x(t):=x(t-\tau)
$$

and the modulation operator,

$$
M_{\nu} x(t):=x(t) e^{j 2 \pi \nu t},
$$

we can rewrite (56) as,

$$
P_{T} \mathcal{N}_{S} Q_{W}=\sum_{m, n} c_{m, n} P_{T} M_{\frac{1}{T}}^{m} T_{\frac{1}{W}}^{n} Q_{W}
$$

where the $c_{m, n}=\hat{S}\left(\frac{m}{T}, \frac{n}{W}\right)$ and $\mathcal{N}_{S}$ is the narrowband channel operator defined in (37). Restating the channel operator in this setting, we can ask what general properties of the operators allow us to express the channel as a double summation of transformed input waveforms. In this section, we determine properties of the operators that are sufficient conditions for the existence of such an expansion. Our goal is to develop an analogous time-scale canonical channel model. That is, in Section 3.6 we propose projections $P$ and $Q$ such that,

$$
P \mathcal{W}_{L} Q=\sum_{m, n} c_{m, n} P D_{a_{0}}^{m} T_{b_{0}}^{n} Q
$$


for some choice of dilation and translation spacing parameters $\left(a_{0}\right.$ and $\left.b_{0}\right)$, where the $c_{m, n}$ depend on $\mathcal{L}$, and $D$ is the dilation operator,

$$
D_{a} x(t):=\frac{1}{\sqrt{|a|}} x\left(\frac{t}{a}\right),
$$

for the wideband channel operator defined in (38).

3.4. Generalization. For the statement of the general theorem, we require the following definition.

DeFINITION 3.1 (paired-up operators). $P$ and $U$ are paired-up operators with generator $e_{0}$ iff,

1. $P$ is an orthogonal projection in $L^{2}(\mathbb{R})$

2. $U$ is unitary in $L^{2}(\mathbb{R})$

3. $P U=U P$

4. $\exists e_{0} \in \operatorname{Ran} P$ s.t. $\left\{U^{m} e_{0}: m \in \mathbb{Z}\right\}$ is an orthonormal basis for Ran $P$

Using two different pairs of paired-up operators, the following theorem gives a sufficient condition for the channel expansion.

THEOREM 3.2. If $(P, U)$ and $(Q, V)$ are both paired-up operators with generator elements $e_{0}$ and $f_{0}$ respectively, $H$ is a bounded operator, and $\exists c_{m, n}$ such that

$$
\sum_{m, n} c_{m, n}\left\langle V^{n+k} f_{0}, U^{l-m} e_{0}\right\rangle=\left\langle H V^{k} f_{0}, U^{l} e_{0}\right\rangle, \quad \forall k, l,
$$

then,

$$
P H Q=\sum_{m, n} c_{m, n} P U^{m} V^{n} Q .
$$

The proof of this theorem can be found in Appendix A and a method for calculating the coefficients $c_{m, n}$ can be found in Appendix B.

3.5. Revisiting time-frequency. The example we have seen so far of the application of this theorem corresponds to the situation

- $\left(P, U, e_{0}\right)=\left(P_{T}, M_{\frac{1}{T}}, \frac{1}{\sqrt{T}} 1_{[0, T]}(t)\right)$

- $\left(Q, V, f_{0}\right)=\left(Q_{W}, T_{\frac{1}{W}}, \sqrt{W} \operatorname{sinc}(W t)\right)$

for the operator $H=\mathcal{N}_{S}$ of the form,

$$
H x(t)=\iint S(\theta, \tau) e^{j 2 \pi \theta t} x(t-\tau) \mathrm{d} \theta \mathrm{d} \tau .
$$

Modulation and translation operators are a natural fit with our channel description, $\mathcal{N}_{S}$, which describes the channel as a (continuous) summation of time and frequency shifts of the input signal. In Appendix B we demonstrate the coefficient calculation procedure for these specific operators. The procedure correctly derives the result $c_{m, n}=\hat{S}\left(\frac{m}{T}, \frac{n}{W}\right)$ where $\hat{S}$ is defined in (57). 
3.6. Time-scale canonical model. We now develop the time-scale canonical characterization. For other possible extensions to time-scale, see the approach in [33, $34,35]$ using wavelet packet modulation.

The Mellin transform (also known as the scale transform) of a signal $x \in L^{2}(0, \infty)$ is defined by

$$
\mathfrak{M} x(\omega):=\int_{0}^{\infty} e^{-j 2 \pi \omega \ln t} x(t) \frac{\mathrm{d} t}{\sqrt{t}}
$$

which represents the composition of two unitary transformations

$$
x(t) \rightarrow e^{t / 2} x\left(e^{t}\right) \stackrel{\mathcal{F}_{t \rightarrow \omega}}{\longrightarrow} \mathfrak{M} x(\omega)
$$

For more information on the Mellin transform and its use in time-frequency analysis we refer the reader to [36]. For the time-scale canonical characterization, we will require the projection operator in the Mellin transform domain

$$
R_{\alpha}:=\mathfrak{M}^{-1} 1_{[-\alpha / 2, \alpha / 2]} \mathfrak{M}
$$

which acts on a function $x \in L^{2}(0, \infty)$ as follows

$$
x(t) \stackrel{\mathfrak{M}}{\longrightarrow} \mathfrak{M} x(\omega) \stackrel{1_{[-\alpha / 2, \alpha / 2]}}{\longrightarrow} 1_{[-\alpha / 2, \alpha / 2]}(\omega) \mathfrak{M} x(\omega) \stackrel{\mathfrak{M}^{-1}}{\longrightarrow} R_{\alpha} x(t)
$$

where $\alpha>0$ defines the cut-off Mellin "frequency". Explicitly, this means

$$
R_{\alpha} x(t)=\int_{0}^{\infty} \frac{1}{\sqrt{t \tau}} \operatorname{sinc}[\alpha(\ln t-\ln \tau)] x(\tau) \mathrm{d} \tau, \quad t>0
$$

Using the characteristic function in the Mellin transform domain,

$$
\Gamma_{0}(\omega)=1_{\left[-\frac{1}{2 \ln a_{0}}, \frac{1}{2 \ln a_{0}}\right]}(\omega)
$$

leads to the scale generator

$$
\gamma_{0}(t)=\left\{\begin{array}{cc}
\frac{1}{\sqrt{\ln a_{0}}} \frac{1}{\sqrt{t}} \operatorname{sinc}\left(\frac{\ln t}{\ln a_{0}}\right) & : \quad t>0 \\
0 & : \quad t<0 .
\end{array}\right.
$$

For further details on the Mellin transform domain and its generators, consult [37].

It can be shown that $\left(P, U, e_{0}\right)=\left(R_{\frac{1}{2 \ln a_{0}}}, D_{a_{0}}, \gamma_{0}(t)\right)$ are paired-up, and thus for the time-scale model, we use the following paired-up operators,

- $\left(P, U, e_{0}\right)=\left(R_{\frac{1}{2 \ln a_{0}}}, D_{a_{0}}, \frac{1}{\sqrt{\ln a_{0}}} \frac{1}{\sqrt{t}} \operatorname{sinc}\left(\frac{\ln t}{\ln a_{0}}\right)\right)$

- $\left(Q, V, f_{0}\right)=\left(Q \frac{1}{b_{0}}, T_{b_{0}}, \frac{1}{\sqrt{b_{0}}} \operatorname{sinc}\left(\frac{t}{b_{0}}\right)\right)$

to decompose the wideband channel corresponding to the operator $H=\mathcal{W}_{\mathcal{L}}$ of the form,

$$
H x(t)=\iint \mathcal{L}(a, b) \frac{1}{\sqrt{|a|}} x\left(\frac{t-b}{a}\right) \mathrm{d} a \mathrm{~d} b
$$


into a discrete double summation,

$$
P \mathcal{W}_{L} Q=\sum_{m, n} c_{m, n} P D_{a_{0}}^{m} T_{b_{0}}^{n} Q
$$

In Appendix B we calculate the coefficients in the time-scale case,

$$
c_{m, n}=\iint \mathcal{L}(a, b) \operatorname{sinc}\left(m-\frac{\ln a}{\ln a_{0}}\right) \operatorname{sinc}\left(n-\frac{b}{a b_{0}}\right) \mathrm{d} a \mathrm{~d} b,
$$

and the canonical time-scale model is then ${ }^{2}$

$$
y(t)=\sum_{m, n} \frac{c_{m, n}}{a_{0}^{m / 2}} x\left(\frac{t-n b_{0} a_{0}^{m}}{a_{0}^{m}}\right)
$$

4. Physical Interpretation of Canonical Models. In [10] the canonical model (77) is obtained by using two sampling results: the classical Shannon sampling formula for bandlimited functions, and a similar sampling result for functions that have finite support in the Mellin transform domain. With the help of these two formulas, [10] obtained a decomposition of the received signal into a series of such as (77) where parameters $a_{0}, b_{0}$ are directly related to transmit signal bandwidth and transmit signal Mellin domain bandwidth. The trouble with such a model is that there are no signals that are perfectly (Fourier) frequency bandlimited and Mellin transform bandlimited (similar to the classical result that there are no time-frequency bandlimited signals except for the trivial zero signal). One can argue that the transmit signal is essentially frequency bandlimited, as well as, Mellin domain bandlimited, and thus a decomposition of type (77) should hold approximately. Furthermore, for a practical application, the infinite series (77) is truncated to a finite number of terms consistent with the finite size of the wideband spreading function $\mathcal{L}$. Thus, another approximation is introduced, so overall one might expect that not much is lost by the initial assumption of joint Fourier frequency - Mellin domain band limitedness.

In contrast, the approach we took in [8] does not suffer from the shortcomings outlined above. This different approach uses all the three players: the sender, the channel, and the receiver. The sender prepares the transmit signal by tailoring some of its properties. That is, the signal is embedded into the range of an orthogonal projection $Q$ (e.g. $Q$ can be an ideal lowpass filter); The channel acts via the operator $H(5)$; and the receiver observes the channel output but in the observation process applies its own projection operator $P$ through measurement, e.g. $P$ is a time cutoff operator. Thus, the entire transmitter-channel-receiver chain is modeled by a "sandwich" of operators $P H Q$ where $P$ and $Q$ are under the user's control, and $H$ is the channel operator. To simplify notation, the transmit signal is assumed to lie already in the range of $Q$, and thus $Q$ often disappears from formulae.

\footnotetext{
${ }^{2}$ It is also possible to reverse the order of application of $T$ and $D$ in (75). In such case, the generated model in fact is more like that in (74) in that the time-shifts are not scaled.
} 
Thus, our basic model for transmitter-to-receiver communication system contains three blocks (see Figure 3):

1. A transmit signal shaper, which is mathematically translated into a projection $Q$; this can be thought of as the last stage of a modulator which, for narrowband communication channels, is either a bandpass filter around the carrier frequency, or a lowpass filter when analysis is done in the base band;

2. A physical channel, mathematically modeled by a linear time-varying system $H$; as such it can be written as in (5);

3. A received signal observation, which again is translated into another projection $P$ at the receiver; typically for memoryless source and channels, this is a time cut-off operator, due to real-time operation constraints.

By changing the transmitter shaping and receiver observation projections, we obtain the different canonical representations. With this interpretation in mind, we can revisit previous models.

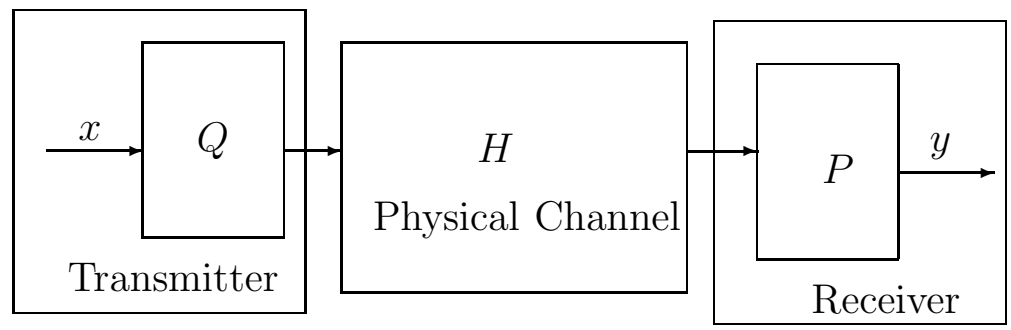

FIG. 3. Our basic model for a communication channel.

The standard rake receiver uses a channel model of type:

$$
y(t)=\sum_{n} h_{n}(t) x\left(t-\frac{n}{W}\right)
$$

which is obtained for $P$ equal to the identity operator (i.e. the entire channel output is available for processing) and for $Q$ equal to the projection onto the space of frequency bandlimited functions. As mentioned before, $x$ is already assumed to be a frequency bandlimited signal, thus $x \in \operatorname{Ran} Q$.

The time-frequency channel model of [5] uses the model (6) which is obtained when $P$ is the time cut-off multiplication by $1_{[0, T]}$ and $Q$ is the ideal lowpass filter.

The time-scale channel model of [8] in (77) uses the ideal Mellin domain lowpass filter as $P$ and the ideal lowpass filter as $Q$. In other words, the channel output is observed through a scale filter defined using the Mellin transform. We now present another canonical model in which the pair of projectors consists of the time cut-off $1_{\left[T_{1}, T_{2}\right]}(t)$ for $P$ and the ideal Mellin domain lowpass filter for $Q$.

4.1. The Frequency-Scale Canonical Model. We now consider a frequencyscale canonical channel characterization based on the translation operators in fre- 
quency and scale. In the frequency-scale model, we restrict ourselves to $x(t)$ defined for $t>0$ and use for the transmitter projection $Q$ the Mellin domain band limiter $R \frac{1}{2 \ln a_{0}}$. Thus, the transmitter transmits scale limited waveforms. We use for the receiver projection $P$ simply a time cut-off

$$
P_{\left[T_{1}, T_{2}\right]} x(t):=1_{\left[T_{1}, T_{2}\right]}(t) x(t)
$$

where $T_{2}>T_{1}>0$ define the receiver observation time horizon. The overall chain of operators then decouples into the series

$$
P H Q \longrightarrow \sum_{m, n} c_{m, n} P_{\left[T_{1}, T_{2}\right]} M_{1 /\left(T_{2}-T_{1}\right)}^{m} D_{a_{0}}^{n} R_{\frac{1}{2 \ln a_{0}}} .
$$

For this model the following theorem gives a decomposition into a series of dilated and frequency shifted versions of the input signal.

Theorem 4.1 (The Canonical Frequency-Scale Channel Model). Assume a timevarying channel $H$ defined by (5). Then for any signal $x$ that is Mellin domain bandlimited to $\left[-\frac{1}{2 \ln a_{0}}, \frac{1}{2 \ln a_{0}}\right]$, i.e. $x \in \operatorname{Ran} Q$,

$$
y(t):=H x(t)=\sum_{m, n \in \mathbb{Z}} c_{m, n} e^{j 2 \pi m \Omega t} \frac{1}{a_{0}^{n / 2}} x\left(\frac{t}{a_{0}^{n}}\right)
$$

for all $T_{1}<t<T_{2}$, where $\Omega=\frac{1}{T_{2}-T_{1}}$,

$$
\begin{aligned}
c_{m, n}= & \frac{1}{\Omega^{2}} e^{-j m \pi \Omega\left(T_{1}+T_{2}\right)} \\
& \int_{-\infty}^{\infty}\left(\int_{0}^{\infty} \hat{\rho}(\omega, a) e^{j \pi \omega\left(T_{1}+T_{2}\right)} \operatorname{sinc}\left(\frac{\omega}{\Omega}-m\right) \operatorname{sinc}\left(\frac{\ln a}{\ln a_{0}}-n\right) \mathrm{d} a\right) \mathrm{d} \omega,
\end{aligned}
$$

and $\hat{\rho}$ is computed in turn from $h(t, \tau)$ through (113).

The convergence in (80) is in the $L^{2}$ sense. The proof of Theorem 4.1 is included in Appendix D. In (80) we see that if we receive scale limited waveforms over a finite time window, that we can decompose the time varying channel into a discrete representation involving a countable sum of weighted scale frequency shifts of the transmitted waveform.

5. Summary and Future Work. Table 5 summarizes the projection and translation operators used to generate the three discrete canonical channel model discussed in this paper. Each of the models can be thought of as sending a transmit waveform through a shaping transmission filter $Q$ and then receiving the signal through a receiving filter $P$. The corresponding discrete channel models are presented in Table 6 . We have presented here a general theory which generates these models based on assumptions on the transmitter and receiver characteristics:

- The time-frequency model arises from:

- frequency-bandlimited transmit waveforms 
TABLE 5

Summary of canonical models of the form $P H Q=\sum_{m, n} c_{m, n} P U^{m} V^{n} Q$.

\begin{tabular}{|c||c|c|c|c|}
\hline model & $P$ & $U$ & $V$ & $Q$ \\
\hline time-frequency & $P_{T}$ & $M_{\frac{1}{T}}$ & $T_{\frac{1}{W}}$ & $Q_{W}$ \\
\hline time-scale & $R_{\frac{1}{2 \ln a_{0}}}$ & $D_{a_{0}}$ & $T_{b_{0}}$ & $Q_{\frac{1}{b_{0}}}$ \\
\hline frequency-scale & $P_{\left[T_{1}, T_{2}\right]}$ & $M_{1 /\left(T_{2}-T_{1}\right)}$ & $D_{a_{0}}$ & $R_{\frac{1}{2 \ln a_{0}}}$ \\
\hline
\end{tabular}

TABLE 6

Summary of canonical models.

\begin{tabular}{|c|c|}
\hline model & characterization \\
\hline time-frequency & $y(t)=\sum_{m, n} c_{m, n} e^{j 2 \pi m t / T} x\left(t-\frac{n}{W}\right)$ \\
\hline time-scale & $y(t)=\sum_{m, n} c_{m, n} \frac{1}{a_{0}^{m / 2}} x\left(\frac{t-n b_{0} a_{0}^{m}}{a_{0}^{m}}\right)$ \\
\hline frequency-scale & $y(t)=\sum_{m, n} c_{m, n} e^{j 2 \pi m t /\left(T_{2}-T_{1}\right)} \frac{1}{a_{0}^{n / 2}} x\left(\frac{t}{a_{0}^{n}}\right)$ \\
\hline
\end{tabular}

- put through a time-frequency (narrowband) channel

- at a time-limited receiver.

- The time-scale model arises from:

- frequency-bandlimited transmit waveforms

- put through a time-scale (wideband) channel

- to a scale-limited receiver.

- The frequency-scale model arises from:

- scale-limited transmit waveforms

- put through a frequency-scale channel

- to a time-limited receiver.

One of the many items for further study is the question of the physical interpretation of the frequency-scale model. In what settings can we envision a channel which imparts a limited range of frequency and scale shifts of an input signal? Perhaps a direct path only model of a wideband sonar signal reflecting off the undulating surface of the ocean with a moving transmitter would impart simultaneously a frequency shift (caused by the frequency of the ocean surface waves) and a scale shift (caused by the change in transmission path length during transmission). Indeed, one main topic of future research is to characterize the channel scenarios which lead to efficient representation in each of the three models.

Further research topics include a full analysis of the two dimensional delay-dilation and Doppler-dilation rake receivers which arise from these canonical models, including an analysis as to which communication scenarios result in performance gains for the two-dimensional rake over conventional receivers. Also, we hope to generalize 
the information theoretic analysis to the delay-dilation and Doppler-dilation rake receivers similar to that which was done for delay-Doppler rake receiver in [5]. Similarly, it would be of interest to develop a canonical time-scale and frequency-scale multiantenna wideband channel model similar to that proposed in [38] for time-frequency channels. Also, [33, 34, 35] introduce wavelet-based channel models; A comparison of these models to the model derived in this work in Section 3 is a topic of future research. Finally, we ask, is there a corresponding underspread/overspread theory (see $[22,39])$ for the time-scale and frequency-scale canonical models?

\section{Appendix.}

A. Proof of Main Theorem. Proof. First we expand $P Q$ using the orthonormal basis and unitary properties of the paired-up operators,

$$
P=\sum_{m}\left\langle\cdot, U^{m} e_{0}\right\rangle U^{m} e_{0}
$$

and

$$
Q=\sum_{n}\left\langle\cdot, V^{n} f_{0}\right\rangle V^{n} f_{0}
$$

we derive,

$$
\begin{aligned}
P Q x & =\sum_{m}\left\langle Q x, U^{m} e_{0}\right\rangle U^{m} e_{0} \\
& =\sum_{m}\left\langle\sum_{n}\left\langle x, V^{n} f_{0}\right\rangle V^{n} f_{0}, U^{m} e_{0}\right\rangle U^{m} e_{0} \\
& =\sum_{m, n}\left\langle x, V^{n} f_{0}\right\rangle\left\langle V^{n} f_{0}, U^{m} e_{0}\right\rangle U^{m} e_{0} .
\end{aligned}
$$

We use this to determine,

$$
\begin{gathered}
P\left(\sum_{m, n} c_{m, n} U^{m} V^{n}\right) Q x=\sum_{m, n} c_{m, n} U^{m} P Q V^{n} x \\
=\sum_{m, n} c_{m, n} U^{m}\left(\sum_{k, l}\left\langle V^{l} f_{0}, U^{k} e_{0}\right\rangle\left\langle V^{n} x, V^{l} f_{0}\right\rangle U^{k} e_{0}\right) \\
=\sum_{m, n, k, l} c_{m, n}\left\langle V^{l} f_{0}, U^{k} e_{0}\right\rangle\left\langle x, V^{-n} V^{l} f_{0}\right\rangle U^{m} U^{k} e_{0} \\
=\sum_{u, s}\left(\sum_{m, n} c_{m, n}\left\langle V^{n+u} f_{0}, U^{s-m} e_{0}\right\rangle\right)\left\langle x, V^{u} f_{0}\right\rangle U^{s} e_{0}
\end{gathered}
$$

where the commuting property of paired-up operators was used in (85a), (84c) was used in moving from (85a) to (85b), and the unitary property of $V$ was used in 
moving from (85b) to (85c). Now, looking to the LHS of (67), we expand using the orthonormal basis and obtain,

$$
\begin{aligned}
\text { PHQx } & =\sum_{s}\left\langle H Q x, U^{s} e_{0}\right\rangle U^{s} e_{0} \\
& =\sum_{s}\left\langle H\left(\sum_{u}\left\langle x, V^{u} f_{0}\right\rangle V^{u} f_{0}\right), U^{s} e_{0}\right\rangle U^{s} e_{0} \\
& =\sum_{s, u}\left\langle x, V^{u} f_{0}\right\rangle\left\langle H V^{u} f_{0}, U^{s} e_{0}\right\rangle U^{s} e_{0} \\
& =\sum_{u, s} h_{u, s}\left\langle x, V^{u} f_{0}\right\rangle U^{s} e_{0} .
\end{aligned}
$$

Given $H$, we then compute,

$$
h_{u, s}:=\left\langle H V^{u} f_{0}, U^{s} e_{0}\right\rangle
$$

which we use to solve,

$$
\sum_{m, n} c_{m, n}\left\langle V^{n+u} f_{0}, U^{s-m} e_{0}\right\rangle=h_{u, s}, \quad \forall u, s
$$

for $c_{m, n}$. These $c_{m, n}$ satisfy (67).

B. Solving the Coefficient Equation. We now discuss the form of the solution to (66). We define

$$
a_{k, l}:=\left\langle V^{k} f_{0}, U^{l} e_{0}\right\rangle
$$

and define

$$
\tilde{c}_{m, n}:=c_{n,-m}
$$

which allows us to express (66) as,

$$
\begin{aligned}
h_{u, s} & =\sum_{m, n} c_{m, n}\left\langle V^{n+u} f_{0}, U^{s-m} e_{0}\right\rangle \\
& =\sum_{m, n}\left\langle V^{u-n} f_{0}, U^{s-m} e_{0}\right\rangle \tilde{c}_{n, m} \\
& =(a \star \tilde{c})_{u, s}
\end{aligned}
$$

where

$$
(a \star \tilde{c})_{u, s}:=\sum_{k, l} a_{u-k, s-l} \tilde{c}_{k, l}=\sum_{k, l} a_{k, l} \tilde{c}_{u-k, s-l}
$$

Expressing $h, a$, and $\tilde{c}$ in the Z-transform domain,

$$
\begin{aligned}
A\left(z_{1}, z_{2}\right) & :=\sum_{k, l} z_{1}^{k} z_{2}^{l} a_{k, l}=\sum_{k, l} z_{1}^{k} z_{2}^{l}\left\langle V^{k} f_{0}, U^{l} e_{0}\right\rangle \\
H\left(z_{1}, z_{2}\right) & :=\sum_{k, l} z_{1}^{k} z_{2}^{l} h_{k, l}=\sum_{k, l} z_{1}^{k} z_{2}^{l}\left\langle H V^{k} f_{0}, U^{l} e_{0}\right\rangle \\
\tilde{C}\left(z_{1}, z_{2}\right) & :=\sum_{k, l} z_{1}^{k} z_{2}^{l} \tilde{c}_{k, l}
\end{aligned}
$$


we can write (91c) as,

$$
H=A \tilde{C}
$$

and solve for $\tilde{C}$

$$
\tilde{C}\left(z_{1}, z_{2}\right)=\frac{H\left(z_{1}, z_{2}\right)}{A\left(z_{1}, z_{2}\right)}
$$

In terms of $c_{m, n}$, this is,

$$
c_{m, n}=Z^{-1}\left(\frac{H\left(z_{1}, z_{2}\right)}{A\left(z_{1}, z_{2}\right)}\right)_{-n, m}
$$

where

$$
Z^{-1}\left(F\left(z_{1}, z_{2}\right)\right)_{m, n}=\int_{0}^{1} \int_{0}^{1} e^{-j 2 \pi \theta_{1} m} e^{-j 2 \pi \theta_{2} n} F\left(e^{j 2 \pi \theta_{1}}, e^{j 2 \pi \theta_{2}}\right) \mathrm{d} \theta_{1} \mathrm{~d} \theta_{2}
$$

We can express (98) as a convolution of coefficients by defining

$$
\hat{A}\left(e^{j 2 \pi \theta_{1}}, e^{j 2 \pi \theta_{2}}\right):=\frac{1}{A\left(e^{j 2 \pi \theta_{1}}, e^{j 2 \pi \theta_{2}}\right)}
$$

and

$$
\hat{a}_{m, n}:=\int_{0}^{1} \int_{0}^{1} e^{-j 2 \pi \theta_{1} m} e^{-j 2 \pi \theta_{2} n} \hat{A}\left(e^{j 2 \pi \theta_{1}}, e^{j 2 \pi \theta_{2}}\right) \mathrm{d} \theta_{1} \mathrm{~d} \theta_{2},
$$

and we can obtain the $c_{m, n}$ using

$$
c_{m, n}=\tilde{c}_{-n, m}=(\hat{a} \star h)_{-n, m} .
$$

Coefficient Calculation. Thus, to calculate the coefficients $c_{m, n}$,

1. calculate $h_{k, l}$ via (87),

2. calculate $a_{m, n}$ via (89),

3. use $a_{m, n}$ to obtain $A\left(e^{j 2 \pi \theta_{1}}, e^{j 2 \pi \theta_{2}}\right)$ via (93),

4. use $A\left(e^{j 2 \pi \theta_{1}}, e^{j 2 \pi \theta_{2}}\right)$ to obtain $\hat{a}_{m, n}$ vua (100) and (101), and

5. use $h_{k, l}$ and $\hat{a}_{m, n}$ to obtain $c_{m, n}$ via (102).

Here, we present the highlights of the coefficient calculation procedure for the time-frequency and time-scale canonical models. For more detailed steps, consult [7].

\section{Example: Time-frequency.}

$$
\begin{gathered}
h_{k, l}=\sqrt{\frac{W}{T}} \iiint 1_{[0, T]}(t) e^{j 2 \pi t\left(\theta-\frac{l}{T}\right)} \operatorname{sinc}(W t-k-W \tau) S(\theta, \tau) \mathrm{d} \theta \mathrm{d} \tau \mathrm{d} t \\
a_{m, n}=\sqrt{\frac{W}{T}} \int_{0}^{T} e^{-j 2 \pi \frac{n t}{T}} \operatorname{sinc}(W t-m) \mathrm{d} t
\end{gathered}
$$


For $\theta_{1}, \theta_{2} \in[0,1]$

$$
\begin{aligned}
& A\left(e^{j 2 \pi \theta_{1}}, e^{j 2 \pi \theta_{2}}\right)=\left\{\begin{aligned}
\sqrt{W T} e^{j 2 \pi W T \theta_{1} \theta_{2}} & : \theta_{1} \in\left(0, \frac{1}{2}\right) \\
\sqrt{W T} e^{j 2 \pi W T\left(\theta_{1}-1\right) \theta_{2}} & : \theta_{1} \in\left(\frac{1}{2}, 1\right)
\end{aligned}\right. \\
& \hat{a}_{m, n}=\frac{1}{\sqrt{W T}} \int_{0}^{1} e^{-j 2 \pi \theta_{2} n} \operatorname{sinc}\left(W T \theta_{2}+m\right)=\frac{1}{W T} a_{-m, n} \mathrm{~d} \theta_{2} \\
& c_{m, n}=\iint S(\theta, \tau) e^{j \pi(T \theta+m)} \operatorname{sinc}(T \theta+m) \operatorname{sinc}(n+W \tau) \mathrm{d} \theta \mathrm{d} \tau
\end{aligned}
$$

which are precisely the coefficients in (58).

\section{Example: Time-scale.}

$$
\begin{aligned}
h_{u, s}= & \frac{1}{\sqrt{b_{0} \ln a_{0}}} \\
& \iint \frac{1}{\sqrt{|a|}} \mathcal{L}(a, b)\left(\int_{0}^{\infty} \frac{1}{\sqrt{t}} \operatorname{sinc}\left(\frac{t-b}{a b_{0}}-u\right) \operatorname{sinc}\left(\frac{\ln t}{\ln a_{0}}-s\right) \mathrm{d} t\right) \\
\text { 8) } & a_{m, n}=\sqrt{\frac{1}{b_{0} \ln a_{0}}} \int_{0}^{\infty} \frac{1}{\sqrt{t}} \operatorname{sinc}\left(\frac{t}{b_{0}}-m\right) \operatorname{sinc}\left(\frac{\ln |t|}{\ln a_{0}}-n\right) \mathrm{d} t .
\end{aligned}
$$

For $\theta_{1}, \theta_{2} \in\left[-\frac{1}{2}, \frac{1}{2}\right]$, in distributional sense,

$$
\begin{aligned}
& A\left(\theta_{1}, \theta_{2}\right)=\sqrt{\frac{1}{b_{0} \ln a_{0}}} b_{0}^{\frac{1}{2}+j 2 \pi \frac{\theta_{2}}{\ln a_{0}}} \int_{0}^{\infty} t^{-\frac{1}{2}+j 2 \pi \frac{\theta_{2}}{\ln a_{0}}} e^{j 2 \pi \theta_{1} t} \mathrm{~d} t \\
& \hat{a}_{m, n}=\sqrt{\ln a_{0}} \int_{-\frac{1}{2}}^{\frac{1}{2}} \int_{-\frac{1}{2}}^{\frac{1}{2}} \frac{b_{0}^{-j 2 \pi \frac{\theta_{2}}{\ln a_{0}}} e^{-j 2 \pi \theta_{1} m} e^{-j 2 \pi \theta_{2} n}}{\int_{0}^{\infty} t^{-\frac{1}{2}+j 2 \pi} \frac{\theta_{2}}{\ln a_{0}} e^{j 2 \pi \theta_{1} t} \mathrm{~d} t} \mathrm{~d} \theta_{1} \mathrm{~d} \theta_{2} \\
& c_{m, n}=\iint \mathcal{L}(a, b) \operatorname{sinc}\left(m-\frac{\ln a}{\ln a_{0}}\right) \operatorname{sinc}\left(n-\frac{b}{a b_{0}}\right) \mathrm{d} a \mathrm{~d} b .
\end{aligned}
$$

C. The Equivalence between (5) and (10). In this section we obtain the correspondence relations between the two forms (5) and (10) of a general time-varying linear system when input signal are supported on positive time domain, and the observation is restricted to a positive time horizon.

Consider first the input-output relationship given by (5). For positive time supported input signals, the output is given by

$$
y(t)=\int_{0}^{\infty} h(t, t-\tau) x(\tau) \mathrm{d} \tau
$$


We change the integration variable $\tau \rightarrow \frac{t}{a}$, and since we have a positive time horizon, i.e. $t>0$, we obtain:

$$
y(t)=\int_{0}^{\infty} h\left(t, t-\frac{t}{a}\right) x\left(\frac{t}{a}\right) \frac{t}{a^{2}} d a .
$$

Now denote $\rho(t, a)=\frac{t}{a \sqrt{a}} h\left(t, t-\frac{t}{a}\right)$, and $\hat{\rho}(\omega, a)$ its Fourier transform with respect to $t$. Then the inverse Fourier transform allows us to write

$$
y(t)=\int_{-\infty}^{\infty}\left(\int_{0}^{\infty} \hat{\rho}(\omega, a) e^{j 2 \pi \omega t} \frac{1}{\sqrt{a}} x\left(\frac{t}{a}\right) \mathrm{d} a\right) \mathrm{d} \omega
$$

that is (10), where, explicitly,

$$
\hat{\rho}(\omega, a)=\frac{1}{a \sqrt{a}} \int_{0}^{\infty} e^{-j 2 \pi \omega t} t h\left(t, t \frac{a-1}{a}\right) \mathrm{d} t .
$$

For the converse, assume the input-output relationship in given by (10). Then, performing the integration over $\omega$ first we obtain

$$
y(t)=\int_{0}^{\infty} \rho(t, a) \frac{1}{\sqrt{a}} x\left(\frac{t}{a}\right) \mathrm{d} a .
$$

Next we need to change the integration variable $a$ into $\tau=t-\frac{t}{a}$

$$
y(t)=\int_{-\infty}^{t} \rho\left(t, \frac{t}{t-\tau}\right) \sqrt{\frac{t-\tau}{t}} x(t-\tau) \mathrm{d} \tau
$$

which is exactly (5) with

$$
h(t, \tau)=1_{t>\tau}(t) \sqrt{\frac{t-\tau}{t}} \int_{-\infty}^{\infty} e^{j 2 \pi \omega t} \hat{\rho}\left(\omega, \frac{t}{t-\omega}\right) \mathrm{d} \omega
$$

where $1_{t>\tau}(t):=1_{[\tau, \infty)}(t)$.

D. Proof of Theorem 4.1. We follow the recipe proposed in Appendix B. The two sets of paired-up operators and generators are:

$$
\left(P=1_{\left[T_{1}, T_{2}\right]}, M_{\Omega}, e_{0}(t)=\sqrt{\Omega} 1_{\left[T_{1}, T_{2}\right]}(t)\right)
$$

and

$$
\left(Q=\mathfrak{M}^{-1} 1_{\left[-\frac{1}{2 \ln a_{0}}, \frac{1}{2 \ln a_{0}}\right]} \mathfrak{M}, D_{a_{0}}, f_{0}(t)=\sqrt{\frac{1}{t \ln a_{0}}} \operatorname{sinc}\left(\frac{\ln t}{\ln a_{0}}\right) 1_{t>0}(t)\right) .
$$

First we need to compute $h_{k, l}$ and $a_{m, n}$. We have:

$$
\begin{aligned}
h_{k, l} & =\left\langle H D_{a_{0}}^{k} f_{0}, M_{\Omega}^{l} e_{0}\right\rangle \\
& =\frac{1}{\sqrt{\ln a_{0}}} \int_{-\infty}^{\infty}\left(\int_{0}^{\infty} \hat{\rho}(\omega, a)\left(\int_{T_{1}}^{T_{2}} \frac{1}{\sqrt{t}} e^{j 2 \pi t(\omega-l \Omega)} \operatorname{sinc}\left(\frac{\ln t / a}{\ln a_{0}}-k\right) \mathrm{d} t\right) \mathrm{d} a\right) \mathrm{d} \omega
\end{aligned}
$$




$$
a_{m, n}=\left\langle D_{a_{0}}^{m} f_{0}, M_{\Omega}^{n} e_{0}\right\rangle=\frac{1}{\sqrt{\ln a_{0}}} \int_{T_{1}}^{T_{2}} \frac{1}{\sqrt{t}} e^{-j 2 \pi \Omega n t} \operatorname{sinc}\left(\frac{\ln t}{\ln a_{0}}-m\right) \mathrm{d} t
$$

Next we compute $A\left(z_{1}, z_{2}\right)=\sum_{m, n} a_{m, n} z_{1}^{m} z_{2}^{n}$, at $z_{1}=e^{j 2 \pi \theta_{1}}, z_{2}=e^{j 2 \pi \theta_{2}}$ for $\theta_{1} \in\left[-\frac{1}{2}, \frac{1}{2}\right], \theta_{2} \in[0,1]$. We obtain:

$$
A\left(e^{j 2 \pi \theta_{1}}, e^{j 2 \pi \theta_{2}}\right)=\sqrt{\frac{\Omega}{\left(\theta_{2}+n_{0}\right) \ln a_{0}}} e^{j 2 \pi \theta_{1} \frac{1}{\ln a_{0}} \ln \frac{\theta_{2}+n_{0}}{\Omega}}
$$

where $n_{0}=n_{0}\left(\theta_{2}\right)$ is the only integer so that $\frac{\theta_{2}+n_{0}}{\Omega} \in\left[T_{1}, T_{2}\right)$. Then

$$
\hat{A}\left(e^{j 2 \pi \theta_{1}}, e^{j 2 \pi \theta_{2}}\right)=\frac{1}{A\left(e^{j 2 \pi \theta_{1}}, e^{j 2 \pi \theta_{2}}\right)}=\sqrt{\frac{\left(\theta_{2}+n_{0}\right) \ln a_{0}}{\Omega}} e^{-j 2 \pi \theta_{1} \frac{1}{\ln a_{0}} \ln \frac{\theta_{2}+n_{0}\left(\theta_{2}\right)}{\Omega}}
$$

which has its Fourier expansion with coefficients $\hat{a}_{m, n}$ given by

$$
\hat{a}_{m, n}=\sqrt{\ln a_{0}} \int_{T_{1}}^{T_{2}} \sqrt{t} e^{-j 2 \pi n \Omega t} \operatorname{sinc}\left(m+\frac{\ln t}{\ln a_{0}}\right) \mathrm{d} t .
$$

Then the coefficients $c_{m, n}$ that solve the equation $a \star \tilde{r}=h$ with $\tilde{r}_{n, m}=c_{m,-n}$ are given by

$$
\begin{aligned}
c_{m, n}= & (\hat{a} \star h)_{-n, m} \\
= & \frac{1}{\Omega^{2}} e^{-j m \pi \Omega\left(T_{1}+T_{2}\right)} \\
& \cdot \int_{-\infty}^{\infty}\left(\int_{0}^{\infty} \hat{\rho}(\omega, a) e^{j \pi \omega\left(T_{1}+T_{2}\right)} \operatorname{sinc}\left(\frac{\omega}{\Omega}-m\right) \operatorname{sinc}\left(\frac{\ln a}{\ln a_{0}}-n\right) \mathrm{d} a\right) \mathrm{d} \omega
\end{aligned}
$$

which is exactly (81).

\section{REFERENCES}

[1] T. Kallath, Measurements on time-variant communications channels, IRE Transactions on Information Theory, 8:5(1962), "S229-S236".

[2] P. A. BELlo, Characterization of randomly time-variant linear channels, IEEE Transactions on Communication Systems, CS-11:4(1963), pp. 360-393.

[3] W. KozeK, On the transfer function calculus for underspread ltv channels, IEEE Transactions on Signal Processing, Special Issue on Advanced Communications, 45:1(1997), pp. 219-223.

[4] T. Kallath, Sampling models for linear time-variant filters., Technical Report "Research Laboratory of Electronics, Technical Report No. 352", Massachusetts Institute of Technology, May 1959. M. S. thesis, M. I. T. Dept. of Electrical Engineering.

[5] A. Sayeed And B. Aazhang, Joint multipath-Doppler diversity in mobile wireless communications, IEEE Transactions on Communications, 47:1(1999), pp. 123-132.

[6] G. Matz, A. Molisch, F. Hlawatsch, M. Steinbauer, and I. Gaspard, On the systematic measurement errors of correlative mobile radio channel sounders. IEEE Transactions on Communications, 50:5(2002), pp. 808-821.

[7] S. RickARD, Time-frequency and time-scale representations of doubly spread channels, $\mathrm{PhD}$ thesis, Princeton University, November 2003. 
[8] R. Balan, H. V. Poor, S. Rickard, And S. Verdú, Time-frequency and time-scale canonical representations of doubly spread channels, in: Proc. European Signal Processing Conf., pages 445-448, September 2004.

[9] Y. Jiang And A. PAPAndreou-Suppappola, Characterization of wideband time-varying channels with multipath-scale diversity, in: Proceedings of IEEE Statistical Signal Processing Workshop, pages 50-53, St. Louis, MO, September 2003.

[10] Y. Jiang and A. Papandreou-Suppappola, Time-scale canonical model for wideband system characterization, in: Proceedings of the IEEE International Conference on Acoustics, Speech, and Signal Processing, March 2005.

[11] A. R. Margetts And P. Schniter, Joint scale-lag diversity in mobile ultra-wideband systems, in: Proceedings of the Thirty-Eighth Asilomar Conference on Signals, Systems and Computers, November 2004.

[12] M. Z. Win AND R. A. Scholtz, Characterization of ultra-wide bandwidth wireless indoor channels: a communication-theoretic view, IEEE Journal on Selected Areas in Communications, 20:9(2002), pp. 1613-1627.

[13] D. Cassioli, M. Z. Win, F. Vatalaro, And A. F. Molisch, Effects of spreading bandwidth on the performance of $U W B$ rake receivers, in: IEEE International Conference on Communications (ICC '03), volume 5, pages 3545-3549, 2003.

[14] L. A. ZAdeh, Time-varying networks, I, Proceedings of the IRE, 49, pp. 1488-1502, October 1961.

[15] L. H. Sibul, L. G. Weiss, and T. L. Dixon, Characterization of stochastic propagation and scattering via Gabor and wavelet transforms, Journal of Computational Acoustics, 2:3(1994), pp. 345-369.

[16] S. Benedetto And E. Biglieri, Principles of Digital Transmission with Wireless Applications, chapter 13: Digital Transmission over Fading Channels, pages 686-724. Kluwer Academic/Plenum Publishers, 1999.

[17] E. Biglieri, J. Proakis, and S. Shamai, Fading channels: Information-theoretic and communications aspects, IEEE Transactions on Information Theory, 44:6(1998), pp. 2619-2692.

[18] J. Proakis, Digital Communications, McGraw-Hill, New York, 1984.

[19] Harry L. Van Trees, Detection, Estimation, and Modulation Theory, Part III, John Wiley \& Sons, New York, 1971.

[20] L. A. ZADEH, Frequency analysis of variable networks, Proceedings of the IRE, 38, pp. 291-299, March 1950.

[21] S. Verdú, Multiuser Detection, Cambridge University Press, New York, 1998.

[22] G. Matz and F. Hlawatsch, Time-frequency transfer calculus of LTV systems, in: TimeFrequency Signal Analysis and Processing, Prentice Hall, 2002.

[23] T. KaIlath, Time-variant communication channels, IEEE Transactions on Information Theory, 9:4(1963), pp. 233-237.

[24] M. L. Fowler And L. H. Sibul, A unified formulation for detection using time-frequency and time-scale methods, in: Twenty-Fifth Asilomar Conference on Signals, Systems and Computers, volume 1, pages 637-642, November 1991.

[25] A. P. Chaiyasena, L. H. Sibul, and A. Banyaga, Wavelet transforms, wideband ambiguity functions and group theoryi in: Twenty-Sixth Asilomar Conference on Signals, Systems and Computers, volume 1, pages 140-144, October 1992.

[26] L. G. Weiss, R. K. Young, And L. H. Sibul, Wideband processing of acoustic signals using wavelet transforms. Part 1. Theory, Journal of the Acoustical Society of America, 96:2(1994), pp. 850-856.

[27] L. H. Sibul, L. G. Weiss, And R. K. Young, Weighted time-frequency and time-scale transforms in reproducing kernel Hilbert spaces, IEEE Signal Processing Letters, 4:1(1997), pp. $21-22$. 
[28] L. Rebollo-Neira and J. Fernandez-Rubio, On wideband deconvolution using wavelet transforms, IEEE Signal Processing Letters, 4:7(1997), pp. 207-209.

[29] L. H. Sibul, M. J. Roan, And K.L. Hillsley, Wavelet transform techniques for time varying propagation and scattering characterization, in: Thirty-Second Asilomar Conference on Signals, Systems and Computers, volume 2, pages 1644-1649, November 1998.

[30] G. B. Giannakis and C. Tepedelenlioglu, Basis expansion models and diversity techniques for blind identification and equalization of time-varying channels, Proceedings of the IEEE, 86:10(1998), pp. 1969-1986.

[31] T. A. Thomas And F. W. Vook, Multi-user frequency-domain channel identification, interference suppression, and equalization for time-varying broadband wireless communications, in: IEEE Sensor Array and Multichannel Signal Processing Workshop, pages 444-448, March 2000.

[32] X. Ma and G. B.Giannakis, Maximum-diversity transmissions over time-selective wireless channels, in: IEEE Wireless Communications and Networking Conference (WCNC2002), volume 1, pages 497-501, March 2002.

[33] M. Doroslovacki And H. FAn, Wavelet-based linear system modeling and adaptive filtering, IEEE Transactions on Signal Processing, 44:5(1996), pp. 1156-1167.

[34] H. Zhang AND H. H. FAn, An indoor wireless channel model based on wavelet packets, in: Thirty-Fourth Asilomar Conference on Signals, Systems and Computers, volume 1, pages 455-459, November 2000.

[35] H. ZhANG, H. H. FAn, AND A. LindSEy, A wavelet packet based model for time-varying wireless communication channels, in: IEEE Third Workshop on Signal Processing Advances in Wireless Communications (SPAWC '01), pages 50-53, March 2001.

[36] L. Cohen, Time-Frequency Analysis, Prentice-Hall, Englewood Cliffs, NJ, 1995.

[37] H. Sundaram, S. D. Joshi, and R. K. P. Bhatt, Scale periodicity and its sampling theorem, IEEE Transactions on Signal Processing, 45(1997), pp. 1862-1865.

[38] A. M. SAYEED, Deconstructing multi-antenna fading channels, IEEE Transactions on Signal Processing, pages 2563-2579, October 2002.

[39] G. Matz and F. Hlawatsch, Time-frequency characterization of random channels, in: TimeFrequency Signal Analysis and Processing, Prentice Hall, 2002. 